\title{
Da sala de espera ao leito: Observação de familiares sobre a Unidade de Terapia
}

\section{Intensiva ${ }^{1}$}

\author{
From the waiting room to the bed: Observation of families on the Intensive Care Unit \\ De la sala de espera a la cama: Observación de las familias en la Unidad de Cuidados Intensivos
}

Recebido: 18/01/2021 | Revisado: 21/01/2021 | Aceito: 25/01/2021 | Publicado: 31/01/2021

\author{
Josiele de Lima Neves \\ ORCID: https://orcid.org/0000-0002-8754-059X \\ Universidade Federal de Pelotas, Brasil \\ E-mail: josiele_neves@hotmail.com \\ Eda Schwartz \\ ORCID: https://orcid.org/000-0002-5823-7858 \\ Universidade Federal de Pelotas, Brasil \\ E-mail: edaschwa@gmail.com \\ Maria Elena Echevarría-Guanilo \\ ORCID: https://orcid.org/0000-0003-0505-9258 \\ Universidade Federal de Santa Catarina, Brasil \\ E-mail: elena_meeg@hotmail.com \\ Lílian Moura de Lima Spagnolo \\ ORCID: https://orcid.org/0000-0003-2070-6177 \\ Universidade Federal de Pelotas, Brasil \\ E-mail: lima.lilian@gmail.com \\ Jéssica Stragliotto Bazzan \\ ORCID: https://orcid.org/0000-0002-8457-134X \\ Universidade Federal de Pelotas, Brasil \\ E-mail: jessica_bazzan@hotmail.com \\ Fernanda Lise \\ ORCID: https://orcid.org/0000-0002-1677-6140 \\ Universidade Federal de Pelotas, Brasil \\ E-mail: fernandalise@gmail.com
}

\begin{abstract}
Resumo
Objetivo: avaliar as observações dos familiares de pacientes internados na Unidade de Terapia Intensiva adulto em relação ao ambiente e ao atendimento prestado pelos profissionais. Metodologia: Estudo descritivo, qualitativo, realizado a partir do pré-teste de um estudo metodológico, de adaptação transcultural do instrumento Family Satisfaction in the Intensive Care Units - 24 para o português do Brasil. Participaram 46 familiares, em dois hospitais do Brasil. Os dados foram coletados através de entrevistas semiestruturada e aplicada a análise temática. Resultados: Elaborou-se duas categorias: "observações dos familiares sobre a sala de espera" e "observações dos familiares durante a visita na UTI". Os familiares percebem a sala de espera como um local desconfortável e destacaram o profissionalismo e a qualidade dos cuidados desenvolvidos pela equipe, porém, identificaram a necessidade de reduzir os ruídos do ambiente. Considerações finais: as observações destacadas evidenciam que os familiares reconhecem as competências dos profissionais e demonstram satisfação com a qualidade do cuidado desenvolvido e da atenção recebida. Além disso, identificam a necessidade de implementar melhorias para favorecer a recuperação dos pacientes.
\end{abstract}

Palavras-chave: Família; Observação; Unidades de terapia intensiva; Satisfação pessoal; Enfermagem.

\begin{abstract}
Objective: to evaluate the observations of family members of patients admitted to the adult Intensive Care Unit in relation to the environment and the care provided by professionals. Methodology: Descriptive, qualitative study, carried out from the pre-test of a methodological study, of cross-cultural adaptation of the instrument Family Satisfaction in the Intensive Care Units - 24 for Brazilian Portuguese. 46 family members participated in two hospitals in Brazil. Data were collected through semi-structured interviews and thematic analysis was applied. Results: Two categories were elaborated: "observations of family members about the waiting room" and "observations of family members during the visit to the ICU". Family members perceive the waiting room as an uncomfortable place and highlighted the professionalism and quality of care developed by the team, however, they identified the need to reduce
\end{abstract}

\footnotetext{
${ }^{1}$ Extraído da dissertação, intitulada "Adaptação transcultural e validação preliminar do instrumento Family Satisfaction with Care in the Intensive Care Unit (FS-ICU 24) para o português do Brasil”, apresentada ao Programa de Pós-Graduação em Enfermagem (PPGEnf) da Universidade Federal de Pelotas (UFPel), no ano de 2015.
} 
ambient noise. Final considerations: the highlighted observations show that the family members recognize the professionals' skills and demonstrate satisfaction with the quality of the care provided and the attention received. In addition, they identify the need to implement improvements to favor the recovery of patients.

Keywords: Family; Observation; Intensive care units; Personal satisfaction; Nursing.

\section{Resumen}

Objetivo: evaluar las observaciones de los familiares de los pacientes ingresados en la Unidad de Cuidados Intensivos de adultos en relación con el entorno y la atención brindada por los profesionales. Metodología: Estudio descriptivo, cualitativo, realizado a partir del pre-test de un estudio metodológico, de adaptación transcultural del instrumento Satisfacción familiar en las Unidades de Cuidados Intensivos - 24 para portugués brasileño. 46 familiares participaron en dos hospitales de Brasil. Los datos se recolectaron mediante entrevistas semiestructuradas y se aplicó análisis temático. Resultados: Se elaboraron dos categorías: "observaciones de familiares sobre la sala de espera" y "observaciones de familiares durante la visita a la UCI". Los familiares percibieron la sala de espera como un lugar incómodo y destacaron la profesionalidad y calidad de la atención desarrollada por el equipo, sin embargo, identificaron la necesidad de reducir el ruido ambiental. Consideraciones finales: las observaciones destacadas muestran que los familiares reconocen las habilidades de los profesionales y manifiestan satisfacción con la calidad de la atención brindada y la atención recibida. Además, identifican la necesidad de implementar mejoras para favorecer la recuperación de los pacientes.

Palabras clave: Familia; Observación; Unidades de cuidados intensivos; Satisfacción personal; Enfermería.

\section{Introdução}

A Unidade de Terapia Intensiva (UTI) é um ambiente de alta complexidade que oferece suporte aos pacientes criticamente graves, com alguma instabilidade hemodinâmica ou que necessitem temporariamente de atenção intensiva para avaliação da evolução do quadro clínico, como em períodos de pós-operatório (Puggina et al., 2014).

A participação da família inicia na sala de espera, por ser o local onde os familiares permanecem por prolongado período, aguardando pela visita e por notícias em relação ao quadro clínico do familiar, passando a ter aquele espaço como uma extensão da sua casa. Nesse contexto, o enfermeiro intensivista tem competência para intervir, realizar educação em saúde e promover a integração, aproximando o familiar do ambiente da UTI para que conheça, integre e se habitue com suas práticas e rotinas (Moraes, Pegoraro, \& Borges, 2017).

Neste processo, as famílias adentram um meio desconhecido, muitas vezes percebido como hostil, repleto de um aparato tecnológico complexo, que pode provocar alterações emocionais, pois, se sentem vulneráveis em decorrência dos sentimentos de dúvidas, medo e insegurança. Esses sentimentos e observações são provocadas pela instabilidade da condição de saúde do familiar, informações inconsistentes, falta de sensibilidade por parte dos profissionais ao comunicar as intercorrências que possam provocar atrasos nos horários de visitas (Backes, Erdmann, \& Buscher, 2015).

A UTI não pode ser vista como um setor que implementa apenas tecnologias duras, em que impera o distanciamento entre a tríade paciente, profissional e família, mas sim como promotora de um movimento de acolhida por parte dos profissionais. Durante a internação na UTI, faz-se necessário considerar a individualidade do sujeito, aproximar a família da vivência desse processo que envolve a escuta ativa e a compaixão por parte dos profissionais.

Embora o impacto negativo que essa experiência possa causar, é importante minimizar as visitas restritivas e promover a aproximação entre familiar e paciente, uma vez que isso pode contribuir para o equilíbrio emocional, permitindo que o familiar se sinta incluído nas tomadas de decisões e nos cuidados realizados. Para tanto, facilitar o entendimento dos familiares sobre a importância da sua participação frente aos cuidados pode promover a otimização do tempo de internação do paciente e qualificar a sua avaliação quanto à percepção em relação aos ambientes que compreendem a UTI.

Contudo, objetivou-se a avaliar as observações dos familiares de pacientes internados na Unidade de Terapia Intensiva adulto em relação ao ambiente e ao atendimento prestado pelos profissionais. 


\section{Metodologia}

Trata-se de um estudo descritivo, qualitativo, que integra a pesquisa maior intitulada "Adaptação transcultural e validação preliminar do instrumento Family Satisfaction with Care in the Intensive Care Unit (FS-ICU 24) para o português do Brasil". Sendo analisado o conteúdo das respostas de três questões abertas relacionadas com a satisfação de familiares que vivenciaram a rotina de visitas na UTI, as questões não poderiam ser pontuadas no instrumento com abordagem quantitativa ao qual se propunha. A riqueza de informações e a importância em divulgar as opiniões/observações dos familiares complementam a avaliação deles em relação ao tratamento dos seus pacientes na UTI.

O método qualitativo permite que a coleta de dados ocorra por meio de entrevistas com questões abertas, neste tipo de estudo é importante a interpretação dos dados, por parte do pesquisador, com suas opiniões sobre o fenômeno em estudo (Pereira, Shitsuka, Parreira, \& Shitsuka, 2018).

O estudo foi realizado com familiares de pacientes que estiveram internados na UTI no período de julho a setembro de 2015, a partir do pré-teste de um estudo metodológico que teve como enfoque o processo de adaptação transcultural do FSICU (24).

Em conformidade com a recomendação da literatura para a etapa do pré-teste do FS-ICU (24), formaram parte da amostra de 30 a 40 participantes que atendessem aos critérios de inclusão e exclusão do estudo (Beaton, Bombardier, Guillemin, \& Ferraz, 2000; Pasquali, 2013). Formava parte da estrutura do instrumento em processo de validação, três questões abertas, na qual os participantes poderiam se expressar livremente em relação ao conteúdo do instrumento testado e de aspectos considerados relevante. A vantagem de entrevistar os participantes é a possibilidade de obter maior taxa de respostas, assim como permitir ao entrevistador a observação/avaliação de possíveis dificuldades de compreensão dos itens e oportuniza o registro de itens que possam não ser compreendidos pelos entrevistados (Polit \& Beck, 2011).

O instrumento FS-ICU (24) possui 24 itens distribuídos em duas subescalas que avalia a satisfação com o atendimento de forma geral (Parte 1) e a satisfação em relação à tomada de decisão nos cuidados prestados aos pacientes atendidos em UTI (Parte 2) (Wall, Engelberg, Downey, Heyland, \& Curtis, 2007). No final deste instrumento constam três questões que foram analisadas neste estudo, nessas os familiares descreveram suas observações sobre o atendimento pelos profissionais na UTI, são elas: $1^{\text {aa }}$ ) Você teria alguma sugestão sobre como melhorar o cuidado na UTI? Se sim, por gentileza descreva; $2^{\mathrm{a}}$ ) Você tem algum comentário sobre as coisas que foram bem feitas no atendimento na UTI? e $3^{\text {a }}$ ) Descreva qualquer comentário ou sugestão que você julgue ser útil para a equipe da UTI deste hospital.

Os participantes da pesquisa foram familiares de pacientes que estiveram internados na UTI e os critérios de inclusão utilizados foram ser familiar de paciente que esteve no mínimo dois dias internados na UTI - podendo ser mais de um familiar por paciente; ter mais de 18 anos; falar e compreender o idioma português; e ter realizado no mínimo três visitas ao seu familiar enquanto internado na UTI. Como critérios de exclusão: não apresentar condições emocionais e cognitivas. A todos os familiares que concordaram em participar foi solicitada a assinatura do Termo de Consentimento Livre e Esclarecido. Assim, com a conclusão do pré-teste a Versão Português Consenso Final (FS-ICU-24-VPCF) tornou-se viável para ser aplicada na realidade brasileira e testada quanto às propriedades psicométricas (Neves et al., 2018).

O estudo foi desenvolvido em Unidades de terapia intensiva de dois hospitais de referência localizados na região sul do Brasil, totalizando 20 leitos. Os familiares foram entrevistados após a alta da UTI, em salas reservadas, disponibilizadas pelos hospitais e, com aqueles familiares de pacientes que receberam alta hospitalar ou evoluíram para óbito, a entrevista se deu em domicílio.

A duração média da entrevista foi de 20 minutos. A coleta dos dados foi realizada pela primeira autora e por entrevistadores treinados para a proposta desta pesquisa que receberam um manual com informações para esclarecer que por ser um pré-teste de um instrumento em processo de validação, deveria ser registrado quaisquer dificuldades de entendimento 
do participante no momento da aplicação.

Para a interpretação dos dados provenientes das entrevistas, foi realizada análise temática (Braun \& Clarke, 2006), que preconiza a análise a partir de cinco estágios. No estágio um, houve a transcrição dos dados, leitura e releitura dos dados, apontamento de ideias iniciais, no estágio dois, iniciou-se a codificação sistemática dos dados em todo o conjunto de dados. Esta codificação foi realizada por meio de cores, identificando em cada depoimento ideias em comum para a codificação. No estágio três ocorreu o agrupamento de códigos em temas potenciais; no estágio quatro, a revisão dos temas, gerando um mapa temático de análise. No estágio cinco, foram nomeados os temas e no estágio seis, foi realizada a análise final dos extratos selecionados, produzindo um relatório acadêmico de análise.

De forma a preservar o anonimato dos participantes, na apresentação dos resultados, eles foram identificados pela letra "F" de "FAMILIAR", seguido do número arábico que identifica a ordem de realização das entrevistas (Exemplo: F1, $\mathrm{F} 2 \ldots)$.

A pesquisa respeitou os aspectos éticos preconizados pela resolução no 466/2012 do Conselho Nacional de Saúde (Brasil, 2012), sendo aprovado pelo Comitê de Ética em Pesquisa (CEP) sob parecer n ${ }^{\circ} 1.104 .124$ em 15 de junho de 2015, com Certificado de Apresentação para Apreciação Ética (CAAE) sob o no 43592615.2.0000.5316.

\section{Resultados}

Participaram do estudo 46 familiares, sendo 30 (65\%) do sexo feminino. As idades variaram entre 16 e 73 anos. Em relação aos anos completos e aprovados de estudo 25 (54\%) tinham mais de dez anos, dois (4\%) relataram não terem estudado e 19 (41\%) estudaram menos de dez anos. Quanto ao número de visitas apenas um familiar informou passar o tempo integral, enquanto que dos demais variou entre três a 94 visitas, cada uma com duração aproximada de 30 minutos, três vezes ao dia, podendo ter seu tempo reduzido em virtude de intercorrências com algum paciente.

Este estudo demonstra diferentes olhares desmembrando-se em momentos distintos e importantes para os cuidados em terapia intensiva, assim, após análise temática os dados foram agrupados em duas categorias, denominadas: Observações dos familiares sobre a sala de espera e Observações dos familiares durante a visita. Tais categorias foram subdivididas ao longo do texto, para melhor compreensão dos resultados.

\section{Observações dos familiares sobre a sala de espera}

As observações dos familiares se moldam diante daquilo que eles vivenciam no dia a dia, durante a internação, de seu ente na UTI, associado as suas interações com a equipe e com o ambiente em que foram inseridos momentaneamente. Assim, estas prospecções podem variar de acordo com sua experiência nesta condição.

Dentro desta categoria, os relatos permitiram apresentar duas subcategorias: Percepção sobre o conforto da sala de espera e a atenção dispensada pelos profissionais durante a espera pela visita.

\section{Conforto da sala de espera}

O período em que a família aguarda pela visita é um momento de muita ansiedade para os membros, pois é esse o período que antecede o reencontro ao familiar, na maioria, em estado clínico crítico, pode ser desencadeador de cansaço físico e estresse psicológico. Deste modo, os familiares fizeram observações sobre a sala de espera e sua comodidade.

F08: Colocar cadeiras para os familiares na sala de espera;

F44: Colocar cadeiras para os familiares aguardarem o acesso à UTI;

F10: Melhora na comodidade com o local de espera; 
F29: a sala de espera deveria ser maior e mais confortável;

F31: Sala de espera é desconfortável;

F37: Diminuir a quantidade de familiares na sala de espera. O ideal/preconizado eram três pessoas e em alguns momentos tinha uma família inteira.

A partir das observações/sugestões dos familiares, reconhece-se a necessidade de orientar a família em relação ao fluxo das visitas na UTI, solicitar a compreensão para que não ocorra aglomerações de pessoas na sala, no mesmo horário. Ainda, quando possível, disponibilizar, chás e ou água, cadeiras, ar-condicionado, televisão, jornais e outros instrumentos promotores de conforto, para possibilitar o descanso e a sensação de bem-estar dos familiares na sala de espera.

\section{Atenção dispensada pelos profissionais durante a espera pela visita}

Enquanto os familiares aguardam a visita a seus entes internados, o tratamento e os cuidados a eles continuam a serem realizados, muitas vezes, podendo vir acontecer intercorrências e/ou óbitos nesse período, o que pode desencadear atrasos no horário pré-definido para a entrada das visitas à UTI. Deste modo, pode vir a modificar a rotina da UTI e a atenção dos profissionais aos familiares enquanto eles aguardam a visita.

F6: Não retirar os pacientes em óbito no horário da visita;

F29: O horário de visita deveria ser respeitado;

F12: Muito tempo aguardando pela visita na sala de espera;

F27: O tempo de espera na UTI ser muito demorado;

F29: A equipe poderia agilizar o transporte, melhor a comunicação entre a UTI e a enfermaria.

A rigidez nos horários de visitas na UTI e o reduzido tempo disponibilizado são fatores que interferem na qualidade da atenção do serviço da UTI às famílias. Além desses, respeitar a vontade da família em permanecer com seu ente querido, mesmo em casos de óbitos. Bem como, a necessidade de melhorar a comunicação com a equipe que realiza o transporte e o preparo do novo leito na enfermaria, para que o tempo desse processo seja o mais breve possível.

\section{Observações dos familiares durante a visita}

Nesta categoria, observou-se a necessidade de construir duas subcategorias que refletem as observações dos familiares durante o momento da visita: o ambiente da UTI e a atenção dispensada pelos profissionais aos pacientes e familiares.

Durante a rápida visita aos pacientes internados na UTI, os familiares podem observar vários aspectos em relação ao ambiente da UTI e a atenção dispensada pela equipe multiprofissional.

\section{O ambiente da UTI}

Na percepção dos familiares, existe a necessidade de redução dos ruídos na UTI, posto que, este é um setor onde estão inúmeros pacientes em estado crítico de saúde, conectados a aparelhos vitais para a sua sobrevivência, os quais emitem ruídos, que somados aos diálogos dos profissionais, podem se tornar inconvenientes para a recuperação dos seus familiares (pacientes).

F14: Deveria ser mais silencioso o local, "melhorar o barulho"; 
F28: Familiar poder visitar um pouco mais, pois algumas pessoas são sensíveis e passam acordadas vendo outras pessoas em piores condições;

F35: Durante a visita foram flexíveis/cordiais, permitiram entrada dos familiares. Sugiro a diminuição de barulhos/ruídos, não apenas das máquinas, mas também dos funcionários;

F40: Ter mais silêncio no ambiente, para que as pessoas consigam descansar.

O ambiente da UTI pode tornar-se hostil quando não proporciona o conforto auditivo no limite estabelecido, nas normas técnicas e o respeito à vontade das famílias para realizar visitas prolongadas, quando julgar necessário.

\section{$O$ atendimento prestado pelos profissionais aos pacientes e familiares}

Familiares também realizaram observações positivas em relação ao atendimento prestado pela equipe de saúde, demonstrando satisfação, concepção de que o paciente foi bem assessorado e desejo de que os atendimentos continuem da forma que estão sendo efetivados.

F2: O atendimento na UTI em relação ao paciente foi muito bom;

F4: Fiquei satisfeito com o atendimento;

F13: Continuar como está o atendimento, pois está muito bom;

F14: O atendimento foi bom e o paciente foi bem assessorado, socorrido a tempo e em nenhum momento houve negligência do cuidado;

F17: Foi bem atendido;

F37: Achei tudo bem feito, todos atenciosos, fui bem atendida, serviço excelente;

F45: Da maneira em que fui tratado na visita. Explicaram como era na UTI.

A percepção sobre o trabalho realizado pela equipe de enfermagem, foi definido como um atendimento de excelência, com referência a prontidão de profissionais, com características como carinho, atenção, amor e responsabilidade no atendimento.

F3: Tanto meu familiar quanto minha família foram bem tratados, os enfermeiros tratavam muito bem, os profissionais todos bem preparados;

F6: Excelente atendimento do corpo da enfermagem;

F9: Atenção da equipe de enfermagem;

F26: Os enfermeiros pareciam estar sempre em prontidão;

F39: O atendimento da enfermagem, todos muito atenciosos. Muito carinho, atenção, amor e responsabilidade dos profissionais.

Destacam-se observações dos familiares associando o atendimento satisfatório e o cuidado empregado dentro do contexto, expressados por eles como 'coisas que foram bem-feitas', a certeza de que o paciente estava sendo bem cuidado, atendendo as necessidades intensivas de cada paciente de maneira singular.

F23: Minha filha foi muito bem cuidada por todos, era o "xodó” da UTI, sempre atenderam as necessidades dos pacientes; 
F30: Acredito que todo o cuidado foi ótimo com pacientes e familiares;

F:34: Excelentes profissionais, eu deixava meu pai naquele local sabendo que estava sendo bem cuidado;

F37: Continuar assim porque é ali que eles precisam de cuidado mesmo;

F1. Mudavam o paciente de posição;

F29: Todos demonstraram carinho com meu familiar.

Pode-se fazer uma interface entre o atendimento e o cuidado, representado nas falas dos familiares pela palavra 'carinho', reconhecido como importante forma de cuidado. Diante das nuances do atendimento na UTI pode oferecer a partir da gravidade do paciente, momento este em que o modo de atendimento, de interação, tanto com o paciente quanto com o familiar, proporciona um serviço humanizado e eficiente.

\section{F1: Preocupação com o bem-estar;}

F13: Carinho que tiveram pelo meu companheiro e por mim;

F22: Boas condutas, esclarecimento diários que ajudaram a ficar a par da situação;

F29: Todos demonstraram carinho com meu familiar.

\section{F32: Fizeram tudo que podiam;}

F38: Tanto meu familiar quanto minha família foram bem tratados, os enfermeiros tratavam muito bem, os profissionais todos bem preparados.

Diante da gravidade da condição de saúde dos pacientes com risco de morte, os profissionais atuam dentro de um contexto em que cada decisão é de extrema importância para o tratamento e prognóstico. Os familiares apontaram a satisfação em relação ao profissionalismo e atenção oferecida pela equipe, em forma de preocupação com o seu bem-estar, nas boas condutas e que em todos os momentos fizeram tudo que poderiam pelos pacientes.

\section{Discussão}

Os familiares dos pacientes internados na UTI tiveram a percepção de que a sala de espera é um local desconfortável. Para eles, a disponibilidade de cadeiras na sala de espera é importante para o estabelecimento do conforto no período que estiverem aguardando a visita. Reforçando, o estudo revela que "ter móveis confortáveis na sala de espera da UTI" é considerado relevante para os familiares. Entende-se que essa afirmação pode estar associada, ao nível de escolaridade dos familiares e ao fácil acesso às informações, o que na maioria das vezes, faz com que eles sejam mais conscientes dos seus direitos.

Estudo realizado com familiares de pacientes internados na UTI revelou a possibilidade de eles aguardarem pela visita no hall da UTI e da equipe flexibilizar as regras de visitação em circunstâncias específicas é positivo (Nunes \& Gabarra, 2017). Isso, devido ao fato dos familiares visitantes terem a oportunidade de organizar seu fluxo de visitas e permanecerem na sala de espera conforme disponibilidade e vontade, pois tornam-se disponíveis para conversar com os profissionais da UTI e fortificar os laços com a equipe.

Uma pesquisa realizada com familiares que aguardavam pelo momento da visita à UTI em um corredor, aponta para as diferenças existentes na estrutura física das UTI's, pois, nem sempre é adequada para receber os familiares. Enfatiza-se a necessidade de uma sala de espera confortável, que melhore a comodidade dos familiares enquanto esperam, além disso, a disponibilidade de uma sala reservada, para a comunicação com a equipe (Nunes \& Gabarra, 2017).

Para atender as necessidades básicas dos familiares, o ambiente da sala de espera precisa ser agradável, limpo, 
privativo, próximo a UTI, equipado com móveis confortáveis, meios de distração, disponibilidade de um local para refeições e com bebedouro. Tendo em vista que alguns visitantes não residem na mesma cidade onde está localizada a UTI e passam a maior parte do tempo de internação de seu ente no hospital (Freitas, Menezes, \& Mussi, 2015) e o conforto é um aspecto que contribui para amenizar o sofrimento da internação (Moerschberger \& Zimath, 2017).

Além desses, outro fator mencionado pelos familiares, foi a capacidade de lotação na sala de espera. Nesse sentido, recomenda-se às equipes técnicas de engenharia consultar a equipe de enfermagem para saber as necessidades de espaços dos ambientes, no momento de projetar e construir os hospitais. Quando houver necessidade de manejar o número de pessoas, recomenda-se que a equipe da unidade trace estratégias, em conjunto com o setor administrativo do hospital, com vistas a organizar a liberação dos familiares da portaria até a sala de espera da UTI, conforme o fluxo dentro do horário estabelecido para visitação, assim, garantindo que o ambiente permaneça harmonioso, humanizado e atenue qualquer impacto emocional que venha a prejudicar o familiar. O cuidado com a família do paciente internado na UTI deve direcionar a equipe de profissionais para um enfoque abrangente, incluindo sensibilidade e atenção (Barth, Weigel, Dummer, Machado, \& Tisott, 2016).

Outro fator que pode causar desconforto emocional ao familiar é quando a visita não ocorre no horário previsto, muitas intercorrências acontecem em uma unidade que atende pacientes gravemente doentes. E, infelizmente, alguns evoluem para óbito pouco antes de iniciar a visita. Neste contexto, cabe salientar que, embora cada instituição tenha suas normas para os cuidados pós morte, incluindo o transporte para um local adequado. Deve-se respeitar o Código de Ética da Enfermagem no que tange a privacidade do paciente em qualquer ciclo vital (Brasil, 2017), destaca-se a importância da utilização de lençóis e biombos, assim como, evitar realizar a transferência do corpo no horário de visita, quando precisar passar pela sala de espera. Dessa forma, além de priorizar o respeito ao paciente evita-se qualquer tipo de desconforto e de sentimentos negativos aos familiares que aguardam a visita.

A demora para entrar na UTI, sem que se obtenha informação e/ou justificativa, gera medo, insegura e estresse no familiar, o que contribui para aumentar a ansiedade e para que os familiares se sintam apreensivos e aterrorizados (Costa, Figueiredo \& Schaurich, 2009). O horário de visita dos familiares é extremamente importante para a recuperação dos pacientes internados na UTI (Barth et al., 2016), porém, muito restrito, com horário pré-estabelecido de início e fim, conforme rotina da unidade (Nunes \& Gabarra, 2017; Costa, Figueiredo, \& Schaurich, 2009).

Deste modo, se por intercorrência ou outros motivos o horário da visita atrasar, é importante que se estabeleça uma comunicação adequada com os familiares. De modo que, esse evento não provoque transtornos para a relação familiar/equipe de enfermagem, necessitando de maior atenção por parte da equipe de saúde, nesse sentido.

Parte-se do ponto em que o horário de visitas é sem dúvida o momento ideal para o desenvolvimento de uma relação terapêutica e interpessoal entre a equipe, o cliente e a família. É o momento em que a família, parte integrante da hospitalização do paciente, se encontra no setor, próximo ao seu ente (Barth et al., 2016). Considerado um fator imprescindível, a participação da família no processo de internação na UTI, tanto para eles quanto para os pacientes, ressaltando que seus membros têm como fonte de apoio uns para com os outros (Nunes \& Gabarra, 2017). Uma vez que o afastamento e a separação podem ser considerados como fator gerador de estresse e insegurança, tanto para o paciente como para a família (Barth et al., 2016).

Outro ponto a ser abordado é a gestão de leitos, ou seja, a transferência do paciente da UTI até a enfermaria, conforme abordado por F29 em sua fala. Sobre isso, destaca-se que um fluxo de leitos eficiente, reduz o tempo de espera da equipe, do paciente e do familiar que aguarda, entre outros benefícios (Raffa, Malik, \& Pinochet, 2017). Assim, novas estratégias são necessárias para manejar a falta de leitos e o fluxo transferência de pacientes, entre as equipes, dentro e fora da UTI. Posto que, quando a taxa de transferência externa não está adequada, isso prejudica também a transferência interna (Winkelmann, Flessa, 
Leisten, \& Kramer, 2008). Deste modo, evidencia-se que, melhorar o fluxo de pacientes em hospitais é uma questão importante na gestão de leitos hospitalares, visto que, com este trabalho, pode-se diminuir o tempo de espera para atendimento e aumentar a capacidade efetiva das unidades de terapia intensiva e internação (Raffa, Malik, \& Pinochet, 2017).

Nesse contexto, destaca-se que o processo está todo interligado, iniciando-se na alta de um paciente e consequente liberação do leito, para o qual virá outro, é repleto de pontos críticos na comunicação interna (equipes), sendo necessária sensibilização dos profissionais que irão transferir o paciente, os que o receberão e a equipe que transportará (Raffa, Malik, \& Pinochet, 2017).

Existem ações que podem promover a melhora na eficiência, dentre elas, pode-se citar a especialização da equipe interna para gerenciar a permanência no hospital, pois, mesmo nos casos em que há disponibilidade de leitos, esses devem ser ocupados de maneira adequada. Além disso, essa equipe tem competência para facilitar a comunicação entre os membros da equipe multiprofissional, no intuito de melhorar o processo de alta ou de transferência (Raffa, Malik, \& Pinochet, 2017).

Em relação ao ambiente da UTI, diante da inflexibilidade das normas que impedem o prolongamento do tempo das visitas, as famílias consideram o tempo de visitas insuficientes (Macedo et al., 2016). As famílias destacaram a necessidade de reduzir os ruídos advindos de equipamento vitais para indicar instabilidades clínicas dos pacientes, ou de diálogos dos membros da equipe multidisciplinar em tom elevado de voz. Esses foram reportados pelos familiares como fatores que impedem essa unidade de ser um lugar tranquilo para a plena recuperação da pessoa em estado grave. O horário de visitas vespertino foi apontado como de maior nível de ruídos (Sampaio Neto et al., 2010).

Para melhorar a qualidade do ambiente, a equipe pode planejar e implementar medidas para reduzir os ruídos para tornar a acústica do ambiente saudável. Para tanto, sugere-se o monitoramento sonoro conforme as normas da Organização Mundial da Saúde (Organização Mundial da Saúde, 2002) e da Associação Brasileira de Normas Técnicas (ABNT, 2000) com a regulagem do volume dos sons emitidos pelos aparelhos de monitorização e suporte a vida para, o limite noturno entre 30 a $40 \mathrm{~dB}$ (decibéis) e diurno de até $45 \mathrm{~dB}$ e a implementação de lembretes da necessidade de silêncio.

A presença da família na UTI pode contribuir para a recuperação do paciente, neste sentido, o seu acolhimento pelos profissionais é fundamental para o alcance desse objetivo. Para tanto, a família do paciente em UTI precisa ser inserida nas estratégias de cuidados desenvolvidos pela equipe (Midega, Oliveira \& Fumis, 2019). Por isso, destaca-se a importância de conhecer as suas expectativas, necessidades e crenças para contribuir com a satisfação da família, aliados a habilidades da equipe em estabelecer comunicação clara quanto aos procedimentos realizados e obtenção de feedback da família sobre o entendimento.

Como limitação destaca-se que as observações dos familiares podem ter sido superficiais, pois nas Unidades de Terapia Intensiva do estudo, o modelo de visita é restritivo, em que a duração diária não ultrapassa três horas. Este estudo contribui para dar visibilidade à família dos pacientes graves, internados na UTI, destaca as observações geradas através do olhar de quem conhece pouco sobre as rotinas desenvolvidas no serviço e, ao demonstrar essas avaliações auxilia para que os profissionais atentem para atender às necessidades da família e, também se doar para satisfazê-las.

\section{Considerações Finais}

A avaliação das observações dos familiares de pacientes internados na Unidade de Terapia Intensiva revelou o reconhecimento das competências dos profissionais e a satisfação com a qualidade do cuidado desenvolvido e da atenção recebida. Além disso, as famílias apontaram a necessidade de implementar melhorias na estrutura física da sala de espera para o conforto dos familiares e medidas que promovam a redução dos ruídos provocados por equipamentos e pela equipe da unidade, para favorecer a recuperação dos pacientes.

Destaca-se como contribuições à prática do enfermeiro intensivista, a necessidade de atenção ao ambiente da sala de 
espera, redução dos ruídos na UTI, bem como, a validade da obtenção do feedback das famílias dos pacientes, para a partir desses, planejar as intervenções necessárias para melhorar a qualidade da atenção.

Considera-se como limitação deste estudo que as observações dos familiares podem ter sido de vivências superficiais, pois ainda impera a prática de visitas restritivas, com tempo limitado de contato com o familiar, muitas vezes, com desvio de atenção para outros pacientes em estados mais graves de saúde, o que dificulta uma avaliação consistente sobre o ambiente e a atenção dos profissionais.

Sugere-se a realização de outros estudos com o uso de instrumentos validados para avaliar a satisfação das famílias de pacientes que estiveram internados na UTI, como estratégia para identificar os aspectos observados. Assim como, o desenvolvimento de estudos comparativos entre as observações de familiares que participam de visitas restritivas e ampliadas.

\section{Agradecimentos}

À Coordenação de Aperfeiçoamento de Pessoal de Nível Superior (CAPES) pela bolsa de doutorado da primeira e última autora. Ao Conselho Nacional de Desenvolvimento Científico e Tecnológico (CNPq) pela bolsa de produtividade em pesquisa da segunda autora.

\section{Referências}

Associação Brasileira de Normas Técnicas. (2000). NBR 10151: 2000 - Acústica: avaliação do ruído em áreas habitadas, visando o conforto da comunidade: procedimento. ABNT/CB-02 - Comitê Brasileiro de Construção Civil. http://www.sema.df.gov.br/wp-conteudo/uploads/2017/09/NBR-10151-de-2000.pdf

Backes, M. T. S., Erdmann, A. L., \& Buscher, A. (2015). The living, dynamic and complex environment care in intensive care unit. Rev Latino-Am. Enfermagem, 23(03): 411- 418. http://dx.doi.org/10.1590/0104-1169.0568.2570

Barth, A. A., Weigel, B. D., Dummer, C. D., Machado, K. C., \& Tisott, T. M. (2016). Estressores em familiares de pacientes internados na unidade de terapia intensiva. Rev. bras. ter. intensiva, 28(3), 323-329. https://doi.org/10.5935/0103-507X.20160055

Beaton, D. E., Bombardier, C., Guillemin, F., \& Ferraz, M. B. (2000). Guidelines for the process of cross-cultural adaptation of self-reportmeasures. Spine (PhilaPa1976); 25(24):3186-3191. https://doi.org/10.1097/00007632-200012150-00014

Braun, V., \& Clarke V. (2006). Using thematic analysis in psychology. Qualitative Research in Psychology, 3(2), 77-101. https://doi.org/10.1191/1478088706qp063oa

Conselho Federal de Enfermagem. (2017). Resolução COFEN n564/2017. http://www.cofen.gov.br/resolucao-cofen-no-5642017_59145.html

Conselho Nacional de Saúde. (2012). Resolução no 466, de 12 de dezembro de 2012, no 12, Seção 1. https://bvsms.saude.gov.br/bvs/saudelegis/cns/2013/res0466_12_12_2012.html

Freitas, K. S., Menezes, I. G., \& Mussi, F. C. (2015). Validação da escala de conforto para familiares de pessoas em estado crítico de saúde. Rev. Latino-Am. Enfermagem, 23(4), 660-8. http://dx.doi.org/10.1590/0104-1169.0180.2601

Macedo, A. P. M. C., Mendes, C. M. F. S., Candeias, A. L. S., Sousa, M. P. R., Hoffmeister, L. V., \& Lage, M. I. G. S. (2016). Validation of the Nursing Activities Score in Portuguese intensive care units. Rev Bras Enferm, 69(5), 826-32. http://dx.doi.org/10.1590/0034-7167-2016-0147

Midega, T. D., Oliveira, H. S. B., \& Fumis, R. R. L. (2019). Satisfaction of family members of critically ill patients admitted to a public hospital intensive care unit and correlated factors. Rev bras ter intensiva, 31(2), 147-155. http://dx.doi.org/10.5935/0103-507x.20190024

Moerschberger, M. S., \& Zimath, S. C. (2017). Necessidades e estressores vivenciados por familiares de pacientes politraumatizados internados em Unidade de Terapia Intensiva. Rev. SBPH, 20(1), 122-142. http://pepsic.bvsalud.org/scielo.php?script=sci_arttext\&pid=S1516-08582017000100008\&lng=pt

Moraes, A. S., Pegoraro, R. F., \& Borges, C. S. (2017). Utilização de vídeo explicativo como recurso auxiliar para acolhimento de famílias em visita à UTI. Rev Psicol Saúde, 9(1), 139-151. http://dx.doi.org/10.20435/pssa.v9i1.475

Neves, J. L., Schwartz, E., Echevarría-Guanilo, M. E., Zanetti, A. C. G., Heyland, D., \& Spagnolo, L. M. L. (2018). Cross-cultural adaptation of the Family Satisfaction with Care in the Intensive Care Unit for Brazil. Cienc Cuid Saude, 17(4). http://dx.doi.org/10.4025/cienccuidsaude.v17i4.45069

Nunes, M. E. P., \& Gabarra, L. M. (2017). Percepção de familiares sobre visitas a pacientes e regras em Unidade de Terapia Intensiva. Arquivos de Ciências da Saúde, 24(3), 84-88. https://doi.org/10.17696/2318-3691.24.3.2017.669

Organização Mundial de Saúde. (2002). Le bruit au tavail el le bruit ambient. Aide-mémoire. Genéve: OMS https://apps. who.int/iris/bitstream/handle/10665/39513/9242540722;jsessionid=3ED97DF1A3FCD3BCAD4A86A06BCF6F49?sequence=1

Pasquali, L. (2013). Psicometria: Teoria dos testes na Psicologia e na Educação. Artmed, p.39. 
Research, Society and Development, v. 10, n. 1, e58810112111, 2021

(CC BY 4.0) | ISSN 2525-3409 | DOI: http://dx.doi.org/10.33448/rsd-v10i1.12111

Pereira A. S., Shitsuka D. M., Parreira, F. J., \& Shitsuka, R. (2018). Metodologia da pesquisa científica. UAB/NTE/UFSM. https://repositorio.ufsm.br/bitstream/handle/1/15824/Lic_Computacao_Metodologia-Pesquisa-Cientifica.pdf?sequence=1

Polit, D. F., \& Beck, C. T. (2011). Fundamentos de pesquisa em enfermagem: avaliação de evidências para a prática de enfermagem: Artmed, p. 669.

Puggina, A. C. G, Ienne, A., Carbonari, K., Parejo, L. S., Sapatini, T. F., \& Silva, M. J. P. (2014). Perception of communication, satisfaction and importance of family needs in the Intensive Care Unit. Escola Anna Nery, 18(2):277-283. http://dx.doi.org/10.5935/1414-8145.20140040

Raffa, C., Malik, A. M., \& Pinochet, L. H. C. (2017). O desafio de mapear variáveis na gestão de leitos em organizações hospitalares privadas. Revista de Gestão em Sistemas de Saúde - RGSS, 6(2). http://dx.doi.org/10.5585/rgss.v6i2.298

Sampaio Neto, R. A, Mesquita, F. O. S, Paiva Junior, M. D. S, Ramos, F. F, Andrade, F. M. D, \& Correia Junior, M. A. V. (2010). Noise in the intensive care unit: quantification and perception by healthcare professionals. Rev bras ter intensiva, 22(4), 369-374. http://dx.doi.org/10.1590/S0103-507X2010000400010

Costa, S. C., Figueiredo, M. R. B., \& Schaurich, D. (2009). Humanização em Unidade de Terapia Intensiva Adulto (UTI): compreensões da equipe de enfermagem. Interface - Comunicação, Saúde, Educação, 13(Suppl. 1), 571-580. https://doi.org/10.1590/S1414-32832009000500009

Wall, R. J., Engelberg, R. A., Downey, L., Heyland, D. K., \& Curtis, J. R. (2007). Refinement, scoring, and validation of the Family Satisfaction in the Intensive Care Unit (FS-ICU) survey. Crit Care Med, 35(1), 271- 279. http://dx.doi.org/10.1097/01.CCM.0000251122.15053.50.

Winkelmann, C., Flessa, S., Leisten, R., \& Kramer, A. (2008). Relevance of economic efficiency analysis of decentralized versus centralized reprocessing of patients' beds in a maximum-care hospital. Hygene und Medizin, 33(6). https://www.researchgate.net/publication/29431347 1_Relevance_of_economic_efficiency_analysis_of_decentralized_and_centralized_reprocessing_of_patients'_beds_in_a_maximum-care_hospital 